\title{
Clinical and epidemiological characteristics of children with germ cell tumors: A single center experience in a developing country
}

\author{
Sonay İncesoy-Özdemir ${ }^{1}$, Ulya Ertem² ${ }^{2}$ Gürses Şahin², Ceyhun Bozkurt², Nazmiye Yüksek ${ }^{3}$, \\ Ayşe Ceyda Ören², Eda Balkaya², Afra Alkan ${ }^{4}$ \\ Departments of ${ }^{1}$ Pediatric Oncology, and ${ }^{4}$ Biostatistics, Ylldrrm Beyazıt University, Ankara, Turkey; ${ }^{2}$ Department of \\ Pediatric Oncology, Dr. Sami Ulus Children's Hospital, Ankara, Turkey; ${ }^{3}$ Department of Pediatric Oncology, Zonguldak \\ Karaelmas University, Zonguldak, Turkey. E-mail: sincesoy@yahoo.co.uk \\ Received: 25th March 2017, Revised: 29th May 2017, Accepted: 1st June 2017
}

SUMMARY: İncesoy-Özdemir S, Ertem U, Şahin G, Bozkurt C, Yüksek N, Ören AC, Balkaya E, Alkan A. Clinical and epidemiological characteristics of children with germ cell tumors: A single center experience in a developing country. Turk J Pediatr 2017; 59: 410-417.

Germ cell tumor (GCT) is a rare malignancy accounting for $2-3 \%$ of all pediatric tumors. The overall survival rate of children and adolescents with GCT is more than $80 \%$ after adopting combined therapy. The aim of this study is to review clinical presentation, management, and outcome in a single-center series with extracranial GCT. Clinical characteristics, pathologic presentations, and survival outcomes of 101 children with GCT, treated at our hospital from 1988 to 2011, were analyzed. Sixty-two of patients were female and 39 of them were male. Fifty-eight (57\%) patients had gonadal tumor (24 testicular, 34 ovarian), 43 (43\%) extragonadal. Histologically, teratomas were found most frequently (26 mature, 10 immature), followed by yolk sac tumors (n: 33), mixed malignant tumors (n: 13), embryonal carcinoma (n: 10), disgerminoma (n: 8) and seminoma (n: 1). Twenty-six patients were diagnosed as mature teratoma and we excluded them in the evaluation of staging and survival. Five-year overall and relaps-free survival were $\mathbf{8 0 . 3 \%}$ (mean follow-up time: 215.8 months) and $\mathbf{7 3 . 4 \%}$ (mean follow-up time: 176.2 months), respectively. Five-year survival rates were $93.2 \%$ and $90.2 \%$ in malign GCTs diagnosed after 1999.

Key words: germ cell tumor, treatment, survival, children.

Gonadal and extragonadal germ cell tumors (GCT) are infrequent in childhood, occurring at a rate of 2.4 cases per million children and representing approximately $1 \%$ of cancers diagnosed in persons younger than 15 years. Until recently, the myriad of histologic subtypes and sites of origin and the paucity of cases hindered the standardization of care for such children. ${ }^{1}$ GCTs form an important group of gonadal neoplasms and are also found in a number of extragonadal sites like the mediastinum, pineal and sacrococcygeal regions and retroperitoneum. Although there are considerable differences between GCTs occurring in different anatomical locations they exhibit a remarkable homology, and are considered as a group. ${ }^{2,3}$
GCTs show numerous histologic subtypes. The histologic features of each subtype are independent of presenting clinical characteristics; tumor biology and clinical behavior vary with site of origin, state, and age of the patient. ${ }^{4}$ The introduction of platinum-based chemotherapy resulted in cure rate in excess of $80 \%$, even in advanced disease.

The aim of this study was to review our institutional experience with the clinical and epidemiological characteristics, management and outcome in extracranial GCTs over a period of 23 years.

\section{Materials and Methods}

One hundred and one children with extracranial 
GCT were admitted to our institution between 1988 and 2011. The cut-off date for analysis of these patients was February 2012. Their clinical characteristics, pathologic features, methods of treatment, relaps and survival outcomes were retrospectively evaluated. The tumors were classified according to the Dehner system. ${ }^{5}$ Twenty-six patients were diagnosed as mature teratoma and we excluded them in the evaluation of staging and survival analyses. The Children's Oncology Group (COG) classification system for staging was used. ${ }^{1}$

The study was approved by the Ethics Committee of the Yildırım Beyazıt University (Report number: 2017/08).

The quantitative variables were described using median and range; qualitative variables were described using frequency and percentages. We used chi-square test to examine the relationship between pairs of variables. Overall survival (OS) and relaps free survival (RFS) distributions were estimated by the method of Kaplan-Meier survival analyses. Using the Cox proportional hazards regression model, multivariate analyses were performed to determine the variables that were independently predictive of prognosis. A $p<0.05$ was used for statistical significance.

\section{Results}

During the period of 23 years, 101 patients with extracranial GCTs were admitted to our clinic. Out of the 101 patients, 62 were female and 39 were male. Their median age was 72 months (0-192 months). The vast majority of patients presented with a combination of symptoms and signs; however, the most frequent primary symptoms and signs were abdominal pain and abdominal mass ( $\mathrm{n}$ : 44 patients). Abdominal pain, either acute, cronic, or both was reported in 23 patients. The second most frequent symptom, abdominal distension by the abdominal or pelvic mass, was noted in 21 patients, associated with pain in all. The third important symptom was increase in testicular size with palpable mass. Other symptoms and signs included sacrococcygeal mass, constipation, nausea, vomiting, urinary symptoms and precocious puberty. One patient had acute abdominal condition. The duration of symptoms ranged from 1 day to 6 months, except for one patient who presented with a 2 years (since birth) history of sacrococcygeal

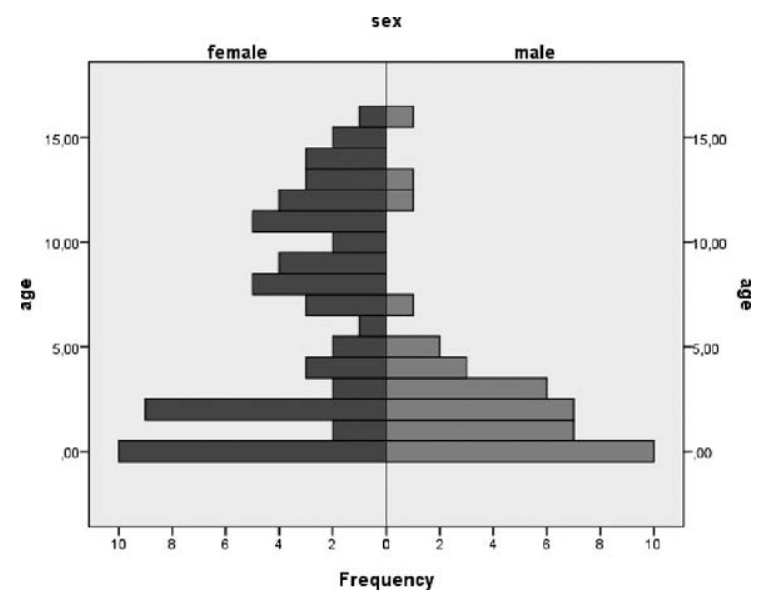

Fig. 1. Gender distribution acccording to age (years).

mass. The clinical presentation of these patients are summarized in Table I.

Figure 1 reveals the bimodal age distribution of GCTs. The first peak was observed during the first 2 years of life both in girls and boys. The second peak started at the age of 8 years in girls and at the age of 12 years in boys. The lowest incidence was found at the age of 5-6 years. Overall, GCTs were more common in girls than in boys (M:F, 1: 1.5). However, among children under 5 years of age, the tumors were more common in boys (M:F, 1.2:1). In the older age group, GCTs were more common in girls (34 females vs. 4 males) (M:F, 0.11:1, $\mathrm{p}<0.001)$. This striking female predominance relates to the high number of ovarian tumors (31 ovarian vs. 4 testicular) in older patients.

\section{Site of origin and diagnostic imaging}

The site of the GCTs varied with genders and age. Fifty-eight (57\%) patients had gonadal tumor (24 testicular, 34 ovarian), 43 (43\%) extragonadal (29 sacrococcygeal, 13 retroperitoneum, 1 neck). Although bilaterality has been reported in gonadal GCTs, no contralateral tumours were identified in this series.

The ovarian tumors were most frequent in the age groups 5 to 10 years and over 10 years. The testis tumors were most frequent in the 1 to 5 years.

Sacrococcygeal and retroperitoneal regions were the most common extragonadal site of origin. Of the extragonadal GCTs, diagnosed during the first year of life, two-thirds $(66 \%)$ ocurred at the sacrococcygeal region. 
Table I. Clinical and Laboratory Characteristics of Patients.

\begin{tabular}{|c|c|}
\hline & n (\%) \\
\hline \multicolumn{2}{|l|}{ Gender } \\
\hline Male & $62(61.3)$ \\
\hline Female & $39(38.6)$ \\
\hline Median age (month) & $72(0-192)$ \\
\hline \multicolumn{2}{|l|}{ Presenting symptoms and signs } \\
\hline Abdominal pain & $23(22.7)$ \\
\hline Abdominal distension & $21(20.7)$ \\
\hline Sacrococcygeal mass & $15(14.8)$ \\
\hline Increase in testicle size & $20(19.8)$ \\
\hline Other & $22(21.7)$ \\
\hline \multicolumn{2}{|l|}{ Site } \\
\hline Ovary & $34(33.6)$ \\
\hline Testis & $24(32.6)$ \\
\hline Extragonadal & $43(42.5)$ \\
\hline \multicolumn{2}{|l|}{ Histopathology } \\
\hline Mature teratoma & $26(25.7)$ \\
\hline Immature teratoma & $10(9.9)$ \\
\hline Yolk-sac & $33(32.6)$ \\
\hline Mixed germ cell tumor & $13(12.8)$ \\
\hline Embriyonal carcinoma & $10(9.9)$ \\
\hline Disgerminoma & $8(7.9)$ \\
\hline Seminoma & $1(0.9)$ \\
\hline \multicolumn{2}{|l|}{ Tumor markers } \\
\hline AFP & $43(57.3)$ \\
\hline B-HCG & $4(5.3)$ \\
\hline AFP and B-HCG & $4(5.3)$ \\
\hline \multicolumn{2}{|l|}{ Stage } \\
\hline I & $19(25.3)$ \\
\hline II & $15(20)$ \\
\hline III & $38(50.6)$ \\
\hline IV & $3(4)$ \\
\hline \multicolumn{2}{|l|}{ Management } \\
\hline Surgery & $33(32.6)$ \\
\hline \multicolumn{2}{|l|}{ Surgery with chemotherapy } \\
\hline BEP & $51(50.4)$ \\
\hline VAC & $17(16.8)$ \\
\hline \multicolumn{2}{|l|}{ Outcome } \\
\hline Alive without disease & $37(49.3)$ \\
\hline Lost to follow up in remission & $13(17.3)$ \\
\hline Lost to follow up with disease & $14(18.6)$ \\
\hline Exitus & $11(10.8)$ \\
\hline
\end{tabular}

AFP: alpha-feto-protein, beta-HCG: human beta chorionic gonadotropin, BEP: bleomycin, etoposide, and cisplatin, VAC: vincristine, actinomycin, cyclophosphamide 
Table II. Number of Tumors According to Site, Histology and Age.

\begin{tabular}{|c|c|c|c|c|c|c|}
\hline \multirow{2}{*}{ Site } & \multirow{2}{*}{$\begin{array}{c}\text { Age } \\
\text { (month) }\end{array}$} & \multicolumn{4}{|c|}{ Histology } & \multirow{2}{*}{ Total } \\
\hline & & Teratoma & Germinoma & $\begin{array}{l}\text { Non-germinom / } \\
\text { non-YST }\end{array}$ & YST & \\
\hline \multirow[t]{4}{*}{ Ovary } & $0-12$ & 0 & 0 & 0 & 0 & 0 \\
\hline & $13-60$ & 0 & 1 & 2 & 0 & 3 \\
\hline & $61-120$ & 6 & 2 & 2 & 4 & 14 \\
\hline & $>120$ & 6 & 5 & 3 & 3 & 17 \\
\hline \multirow[t]{4}{*}{ Testis } & $0-12$ & 1 & 0 & 1 & 3 & 5 \\
\hline & $13-60$ & 3 & 0 & 3 & 9 & 15 \\
\hline & $61-120$ & 0 & 0 & 1 & 0 & 1 \\
\hline & $>120$ & 1 & 1 & 0 & 1 & 3 \\
\hline \multirow[t]{4}{*}{ Extragonadal } & $0-12$ & 14 & 0 & 2 & 3 & 19 \\
\hline & $13-60$ & 7 & 0 & 5 & 9 & 21 \\
\hline & $61-120$ & 0 & 0 & 0 & 2 & 2 \\
\hline & $>120$ & 0 & 1 & 0 & 0 & 1 \\
\hline
\end{tabular}

YST: yolk sac tumor

Abdominal ultrasound was the most common investigation, performed in all patients. Computed tomography was performed for 24 patients. Magnetic resonance imaging was performed only in one patient.

\section{Tumor markers}

Alpha-feto-protein (AFP) and human beta chorionic gonadotropine (B-HCG) levels were normal (based on age) in all patients with mature teratomas (26 patients). We detected high AFP levels in 43 of 75 patients with malignant GCTs, high B-HCG levels in 4 of 75 patients. Both AFP and B-HCG levels were elevated in 4 patients. We found normal AFP

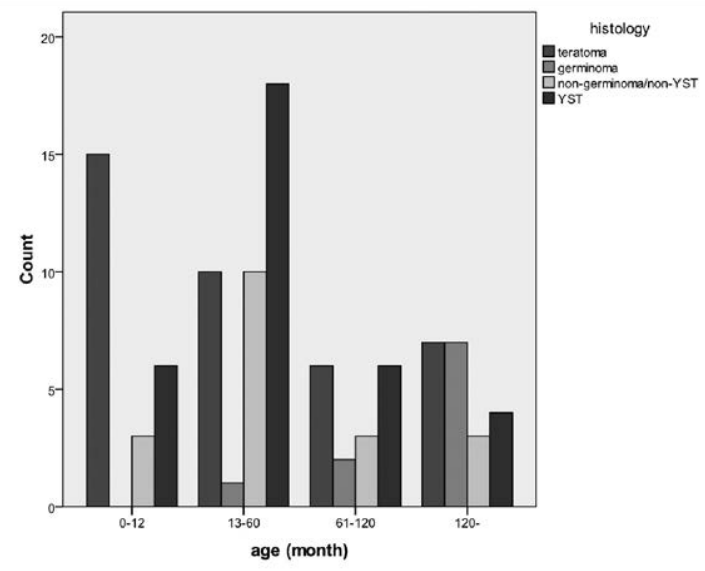

Fig. 2. Age distribution acccording to histology. YSK: yolk sac tumor and B-HCG levels in 17 patients with malignant GCTs (six with immature teratomas, four with embryonal carcinoma, two with seminomas, two with yolk sac tumors, two with mixed malignant tumors and one with disgerminoma). Unfortunately, in two of them tumor markers were measured after surgery (one had stage I disease, another one had stage II disease). Seven patients were not studied for AFP and B-HCG because of socio economic difficulties.

\section{Histology and staging}

Histologically, teratomas were found most frequently (26 mature, 10 immature), followed by yolk sac tumors (n: 33), mixed malignant tumors (n: 13), embryonal carcinoma (n: 10), disgerminoma (n: 8) and seminoma (n: 1).

We observed that the GCT histology in children vary with sex and age groups (Figure 2 and Table II). Figure 2 shows that in infants, the vast majority of GCTs are teratomas. Malignant non-germinomatous GCT, the vast majority of which were yolk-sac tumors, were most common at the age of 13-60 months. Disgerminomas predominantly developed after the age of 5 years. Disgerminomas and teratomas constituted the most frequent histological subtype during adolescence.

Twenty-six patients were diagnosed as mature teratoma and they were excluded from the evaluation of staging and survival. Nineteen 
out of 75 cases $(25.3 \%)$ had stage I, $15(20 \%)$ had stage II, 38 had (50.6\%) stage III and 3 (4\%) had stage IV disease. Additionally, 3 (4\%) out of 38 patients with stage III disease and 3 (4\%) patients with stage IV disease were only operated at other clinics and they admitted to our clinic with relapses.

\section{Treatment}

For all patients with mature and immature teratomas treatment consisted of surgery alone. But, three patients with immature teratomas received chemotherapy because of local and systemic relaps. The first patient with immature teratoma was 29-day-old girl. She was admitted to the clinic with sacrococcygeal mass since birth. At laboratory examination, AFP (based on age) and B-HCG were normal. Complete surgical excision of tumor with coccygectomy was performed. She was close follow-up without any drug therapy every 3 weeks. Two months later, local relapse was identified. Tumor markers were normal. Adjuvant chemotherapy had been administered, consisting of 6 cycles of BEP (bleomycin, etoposide, and cisplatin). However, tumor recurred three months after the chemotherapy ends. AFP was never elevated in this patient. Re-resection was performed, because of local recurrence after systemic chemotherapy. Histologic examination showed that retroconversion of immature teratoma to mature teratoma. The patient had subsequently been lost to follow-up. The second patient, an 8-year-old girl, was referred to our clinic due to abdominal pain and distention for 2 months. CT scan revealed a complex cysticsolid mass, measuring $13 \times 3 \times 14 \mathrm{~cm}$, on the right over and retroperitoneal region. AFP was $52 \mathrm{ng} / \mathrm{ml}(0-8)$, while B-HCG was normal. On exploratory laparotomy, a large right ovarian mass and liver metastasis were identified. Right salpingo-oopherectomy was performed and the resected ovarian tumor. Unfortunatelly, intraoperative rupture of the tumor ocurred during operation. Adjuvant chemotherapy with BEP was administered. The patient completed 6 cycles of BEP. AFP level at the end of treatment was normal. She has maintened complete remission for over 10 years. The third patient, a 4-year-old girl, with a previous diagnosis of sacrococcygeal immature teratoma admitted to our clinic with new liver and pulmonary metastases after 18 months first diagnosis. Five

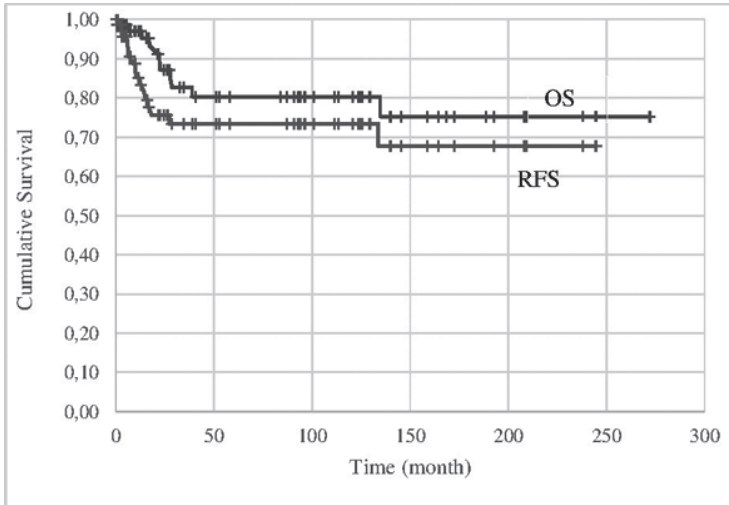

Fig. 3. Overall (OS) and relaps-free survival (RFS) in children with malignant extracranial germ cell tumors, 1988-2011.

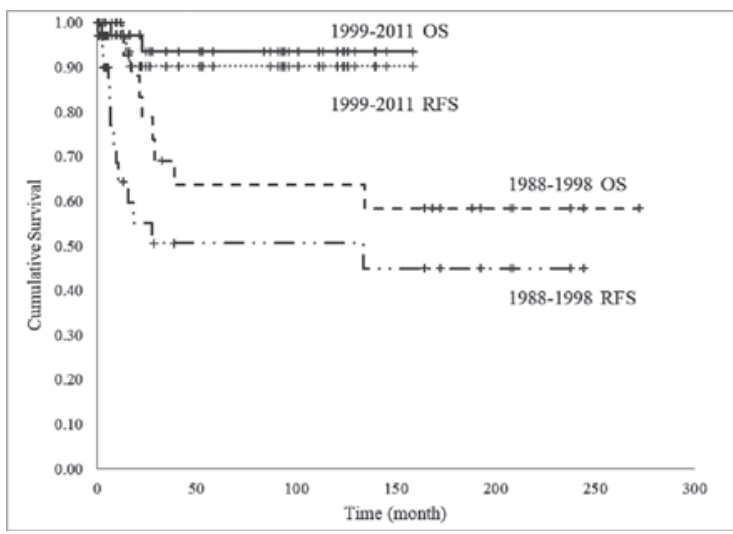

Fig. 4. Overall (OS) and relaps-free survival (RFS) in children with malignant extracranial germ cell tumors, 1988-1998 and 1999-2011.

cycles of chemotherapy were administered, but she was lost to follow-up.

Surgery was considered the sole treatment necessary in $33(32.6 \%)$ patients, surgery + chemotherapy in $66(65.3 \%)$ other patients, and surgery + chemotherapy + radiotherapy in 2 (1.9\%) patients. Tumors were treated with VAC (vincristine, actinomycin, cyclophosphamide) protocol from 1988 to 1994. Cisplatin-based combination chemotherapy was used from 1994 onward. Fifty-one (50.4\%) cases were treated with BEP protocol, 17 (16.8\%) cases with VAC protocol. Only two patients received radiotherapy because of progressive disease.

\section{Outcome}

Thirty-seven $(49.3 \%)$ patients were disease-free at a median follow up of 215.8 months. Eleven patients $(14.7 \%)$ had died from progressive disease ranging between 1 and 38 months 
(median 21 months) after diagnosis. Fourteen patients were lost to follow up at a median follow up of 3 months (ranged, 1-15). Thirteen patients were lost without disease at a median follow up of 28 months (ranged, 12-139).

Sixteen (21.3\%) patients had relapse in 3 to 18 months. The median time for tumor recurrence was 10 months. For the 16 relapses, 9 were locoregional recurrence and 7 were systemic metastases. Six out of them were referred to us for relaps treatment after primary was managed elsewhere. These patients were in the early 1990s, surgical centers are operated, stage 1 evaluated and followed without consultation of pediatric oncology clinic. (Table III).

The 5-year OS and RFS were $80.3 \%$ (mean follow-up time: 215.8 ; 95\% CI: 186.0- 245.7 months) and $73.4 \%$ (mean follow-up time: 176.3; 95\% CI: 147.45- 205.1 months), respectively (Figure 3 ).

We compared two time periods of 23 years (1988-1998 and 1999-2011). The 5-year OS and RFS improved from $63.7 \%$ and $50.5 \%$ in $1988-1998$ to $93.2 \%$ and $90.2 \%$ in $1999-2011$, respectively (p: 0.022; Fig. 4).

\section{Prognostic Factors}

We investigated prognostic factors by univariate and multivariate analysis. Age group, sex, site, histology, stage, tumor marker, and treatment were tested; they did not have a prognostic value. These methods identified patients treated between 1999 and 2011 as having decreased mortality in comparison with those treated in the earlier period (in the multivariate analysis, HR 0.16; 95\% CI: 0.028-0.989; p: 0.049).

\section{Discussion}

Pediatric GCTs are rare tumors. Their overall incidence can be estimated as 0.9/100,000 children up to 15 years. They arise due to variation from normal differentiation of germ cells and include a heterogeneous group of neoplasms with remarkable variability concerning histology and site of presentation: Benign, malignant and immature GCTs can be observed in children and adolescents with different rates, also according to age. ${ }^{3} \mathrm{We}$ have confirmed that the GCT localization and histological subgroups in children vary among genders and age groups. The clinical characteristics found in this study are similar to other national and international studies related to the issue. ${ }^{6-12}$

Median age at diagnosis varied according to tumor site, histology, and sex. In a large population-based cohort study from Germany, including 1,442 cases up to 15 years of age, there was a bimodal age distribution. In this study, the first peak was observed during the first 2 years of life. The second rise of incidence started at the age of 7 years in girls and at the age of 10 years in boys. As the lowest incidence was found at the age of 5-6 years. ${ }^{8}$ In another study from US, incidence peaks were observed before the age of 1 year and from ages 15 to 19 years in both boys and girls. In this study, the incidince of GCTs was smilar in boys and girls in the age group of patients ages birth to 9 years, whereas the incidence was much higher in boys in the group ages 10 to 19 years. ${ }^{6}$ In accordance with other studies, bimodal age distribution was observed in our patients. The first peak was observed during the first 2 years of life both in girls and boys.

Table III. Relapse Patterns and Results.

\begin{tabular}{ll}
\hline & $\mathrm{n}$ \\
\hline Relapse site & 6 \\
Local primary site & 3 \\
Local and intraabdominal & 4 \\
Systemic & 2 \\
Lung & 1 \\
Liver & 6 \\
Lung and liver & 6 \\
Outcome of patients who experienced relapse & 6 \\
Exitus & 4 \\
Lost to follow up & \\
Alive without disease & \\
\hline
\end{tabular}


The second peak started at the age of 8 years in girls and at the age of 12 years in boys. The lowest incidence was found at the age of 5-6 years. Among children under 5 years of age, the tumors were more common in boys $(\mathrm{M}: \mathrm{F}$, 1.2:1), while in the older patients, there was a striking female predominance (M:F, 0.11:1, $\mathrm{p}<0.001)$. This might be attributed to referral of male adolescent patients to adult centers in our country.

Tumor markers are important in the diagnosis, diagnosis of relapse and follow-up. High AFP and beta-HCG levels were detected in $63 \%$ and $11 \%$ of the studied patients, respectively.

Teratomas are the most common GCTs observed in most published series. ${ }^{6,7,9}$ In our series, the most frequently recognized histological types were teratomas and yolk sac tumors. Teratomas constitute the vast majority of GCTs in neonate and that the incidence peak of YST follows approximately 1 year later.

Mature teratomas with normal tumor markers were kept in regular follow-up, but patients having malignant GCTs need follow-up regarding appropriate therapy and survival.

Immature teratomas, although not truly malignant, have been treated as malignant neoplasms in the past because of their potential to recur as malignant tumours. Either immature teratomas or immature teratomas with microscopic foci of yolk sac tumours have been included in the group of malignant GCTs in many series ${ }^{10,12}$; chemotherapy has been given to patients with grade II or III immature teratomas. In our series, three patients with immature teratomas received multi-agent chemotherapy because of relaps and elevated AFP and all remained free of disease at follow-up.

The prognosis in GCTs did not improve until the late 1970s, when the VAC regimen became the standart chemotherapy in GCTs. Since the introduction of platinum-based agents in pediatric chemotherapy regimens, the survival rates for children with malignant GCTs ranging from $75 \%$ to more than $90 \% .{ }^{13-15}$ With accurate staging, complete resection, and chemotherapy for malignant GCTs, patients are expected to have excellent survival rates. In a study frm Turkey, Akyüz et al. ${ }^{11}$ reported OS and EFS in ovarian tumors as $68 \%$ and $57 \%$, respectively.
Feltbower et al. ${ }^{15}$ reported the 5-year survival rates as $93-95 \%$ for gonadal GCT, and $70-75 \%$ for extragonadal GCT. Mann et al. ${ }^{14}$ reported the 5 -year survival rates as $90.9 \%$ and $87.8 \%$. In another recent study from Finland, the overall 5 -year survival was reported to be improved from $63.7 \%$ in $1969-1987$ to $90.7 \%$ in 1988 $2006 .{ }^{9}$ In a more recent large population-based study from Germany, 5-year survival rate was reported as $92 \%$ for gonadal and extragonadal GCTs. ${ }^{10}$

In our series, OS rate $(80.3 \%)$, which is lower than that of other series, could be partially explained by the high number of patients with advanced disease. Additionaly, of 11 patients in our series, 10 died between 1988 and 1998. It was remarkable that within this period, six patients were operated in other surgical centers, followed-up without consulting pediatric oncology departments, and admitted to the clinic with advanced-stage relapse thereafter. Only two of the patients who were diagnosed after 1999 have relapsed so far. It was also considerable that except for one, all other cases had abandoned the treatment within this period. As clearly seen in our study group, survival rates increased from 1988-1998 to 1999-2011 and the number of patients who escaped from treatment decreased over the years.

For extracranial malignant GCTs, the tumour site, age and elevated AFP have been proven to be of importance as prognostic factors. ${ }^{13,16-18}$ Gonadal GCTs have a significantly better prognosis compared to extragonadal tumours. We could not identify site, age and elevated AFP as a prognostic factor. In our study group, the prognosis of malign CCTs has improved over time through advances in treatment. In children with malignant GCTs, survival rates can be improved owing to factors such as advancement in diagnostic and therapeutic techniques, improved supportive care, and a multidisciplinary approach.

In conclusion, some significant differences in clinical features were documented. Among children under 5 years of age, the tumors were more common in boys (M:F, 1.2:1), while in the older patients, there was a striking female predominance (M:F, 0.11:1, $\mathrm{p}<0.001)$. This might be attributed to referral of male adolescent patients to adult centers in our 
country. They were similar to patients in other large pediatric series with respect to sites of origin, age at presentation in relationship to primary site, histology and sex.

Our study has some limitations. This is a retrospective study from a single national institution. Our data has failed to demonstrate any correlation between tumor stage and survival. This can be explained by the very few numbers of patients with stage IV. Unfortunately, our infrastructure capacity to accurately determine stage at dignosis was limited at that time.

However, this series confirms the good overall results that can be achieved in children with malignant GCTs. As parallel to the recent developments across the world, the rate of life expectancy has increased due to the elevation witnessed in general health services in Turkey. The advances in multidisciplinary approach to pediatric oncology care have led to dramatically improved survival for children with GCT in Turkey.

\section{REFERENCES}

1. Cushing B, Perlman EJ, Marina NM, Castleberry RP. Germ cell tumors. In: Pizzo PA, Poplack DG (eds). Principles and Practice of Pediatric Oncology (5th ed). Philadelphia: Lippincot Williams and Wilkins, 2006: 1116-1138.

2. Talerman A. Germ cell tumours. Ann Pathol 1985; 5: 145-157.

3. Cecchetto G. Gonadal germ cell tumors in children and adolescents. J Indian Assoc Pediatr Surg 2014; 19: 189-194.

4. Mann JR, Pearson D, Barrett A. Results of the United Kingdom Children Cancer Study Group's malignant germ cell tumor studies. Cancer 1989; 63: 1657-1667.

5. Dehner LP. Gonadal and extragonadal germ cell neoplasms: teratomas in childhood. In Finegold MJ, Bennington J, eds. Pathology of neoplasia in children and adolescents. Philadelphia: WB Saunders, 1986: 282-312.

6. Poynter JN, Amatruda JF, Ross JA. Trends in incidence and survival of pediatric and adolescent patients with germ cell tumors in the United States, 1975 to 2006. Cancer 2010; 116: 4882-4891.

7. Ablin AR, Krailo MD, Ramsay NK, et al. Result of treatment of malignant germ cell tumors in 93 children: A report of the Children's Cancer Study Group. J Clin Oncol 1991; 9: 1782-1792.

8. Schneider DT, Calaminus G, Koch S, et al. Epidemiologic analyses of 1,442 children and adolescents registered in the German germ cell tumor protocols. Pediatr Blood Cancer 2004; 42: 169-175.
9. Pauniaho SL, Salonen J, Helminen M, et al. Germ cell tumors in children and adolescents in Finland: Trends over 1969-2008. Cancer Causes Control 2014; 25: $1337-1341$.

10. Kaatsch P, Hafner C, Calaminus G, et al. Pediatric germ cell tumors from 1987 to 2011: Incidence rates, time trends, and survival. Pediatrics 2015; 135: e136-e143.

11. Akyüz C, Varan A, Büyükpamukçu N, et al. Malignant ovarian tumors in children: 22 years of experience at a single institution. J Pediatr Hematol Oncol 2000; 22: 422-427.

12. Büyükpamukçu $M$, Varan $A$, Küpeli $S$, et al. Malignant sacrococcygeal germ cell tumors in children: A 30-year experience from a single institution. Tumori 2013; 99: 51-56.

13. Mann JR, Raafat F, Robinson K, et al. UKCCSG's germ cell tumor (GCT) studies: Improving outcome for children with malignant extracranial non-gonadal tumours-carboplatin, etopside, bleomycin are effective and less toxic than previous regimens. Med Pediatr Oncol 1998; 30: 217-227.

14. Mann JR, Raafat F, Robinson K, et al. The United Kingdom Children's Cancer Group's Second Germ Cell Tumor Study: Carboplatin, etoposide, and bleomycin are effective treatment for children with malignant extracranial germ cell tumors with acceptable toxicity. J Clin Oncol 2000; 18: 3809-3818.

15. Feltbower RG, Siller C, Woodward E, et al. Treatment and survival patterns for germ cell tumors among 13- to 24-year olds in Yorkshire, UK. Pediatr Blood Cancer 2011; 56: 282-288.

16. Marina N, London WB, Frazier AL, et al. Prognostic factors in children with extragonadal malignant germ cell tumors: A pediatric intergroup study. J Clin Oncol 2006; 24: 2544-2548.

17. Gobel U, Schneider DT, Calaminus G, et al. Germ-cell tumors in childhood and adolescence. Ann Oncol 2000; 11: 263-271.

18. Baranzelli MC, Kramar A, Bouffet E, et al. Prognostic factors in children with localized malignant nonseminomatous germ cell tumors. J Clin Oncol 1999; 17: 1212-1218. 